\title{
Complicações na utilização de implantes zigomáticos para o tratamento reabilitador de maxilas atróficas: revisão de literatura
}

\author{
Complications in the use of zygomatic implants for the rehabilitation of the atrophic maxilla: a literature review
}

FIAMONCINI, Eduardo Stedile'; GUIMARÃES, Géssyca Moreira Melo de Freitas ${ }^{1}$; ALCALDE, Luis Fernando Azambuja ${ }^{1}$; MELLO, Marina de Almeida Barbosa'; FERREIRA JÚNIOR, Osny'룰 CARVALHO, Paulo Sergio Perri de ${ }^{2}$

1. Mestrando em Cirurgia e Traumatologia Bucomaxilofacial, Faculdade de Odontologia de Bauru, Universidade de São Paulo, Bauru, SP, Brasil

2. Professor Associado da Disciplina de Cirurgia, Faculdade de Odontologia de Bauru, Universidade de São Paulo, Bauru, SP, Brasil.

Endereço para correspondência:

Eduardo Stedile Fiamoncini

Faculdade de Odontologia de Bauru - USP

Disciplina de Cirurgia

Alameda Doutor Octávio Pinheiro Brisolla, 9-75

17012-901 - Bauru - São Paulo - Brasil

E-mail: eduardo.fiamoncini@gmail.com

Recebido: 31.08 .2016

Aceito: 30.06 .2017

\section{RESUMO}

A reabilitação de maxilas atróficas sempre foi um desafio para o cirurgião dentista, e a utilização de implantes zigomáticos tem se popularizado como uma alternativa de tratamento à reconstrução alveolar. O presente trabalho realizou uma revista da literatura buscando complicações trans e pós-cirúrgicas mais comuns relacionadas à cirurgia de implantes zigomáticos. Foi realizada busca na base de dados PubMed por artigos publicados entre 2010 e 2015, sendo encontrados 92 artigos. Destes, foram selecionados estudos que atendessem aos critérios de inclusão e exclusão. Doze dos 92 artigos foram selecionados para revisão, somando um total de 5.425 implantes zigomáticos instalados. Artigos que consideraram apenas as complicações protéticas não foram selecionados. As complicações pós-operatórias relatadas foram: 162 casos de sinusites (2.98\%), 129 de infecção dos tecidos moles (2.37\%), 16 parestesias $(0.29 \%), 21$ fístulas orosinusais $(0.38 \%), 2$ enfisemas subcutâneos (0.036\%), 1 penetração da cavidade orbital (0.018\%), 9 de dor persistente (0.16\%), 5 exposições do implante (0.092\%) e 16 falhas de osseointegração (0.29\%). Artigos de casos clínicos, com um grupo amostral pequeno, relataram ausência de complicações clínicas. O follow-up variou de 1 a 7 anos. A complicação mais frequente é a sinusite maxilar, e a técnica de fixação zigomática pode incorrer em inúmeras complicações se o cirurgião for inexperiente e/ou não tiver bom conhecimento anatômico da região. Faltam estudos com casos controle que discussão as complicações trans e pós-operatórias.

Palavras-chave: Sinusite maxilar. Zigoma. Implantes dentários.

\section{ABSTRACT}

The rehabilitation of atrophic jaws has always been a challenge for the surgeon and the use of zygomatic implants has become a popular alternative treatment to alveolar reconstruction. The present study consists of a literature review seeking trans and postoperative surgical complications that are most commonly related to zygomatic implant surgery. A search was performed in PubMed database for articles published between 2010 and 2015, and 92 articles on the subject were found. Studies were selected based on the inclusion and exclusion criteria. Twelve of the 92 articles were selected for review, with a total of 5.425 zygomatic implants. Articles that only considered prosthetic complications were not selected. The reported post-operative complications were 162 cases of sinusitis (2.98\%), 129 soft tissue infection (2.37\%), $16(0.29 \%), 21$ orosinusais fistulae $(0.38 \%) 2$ subcutaneous emphysema $(0.036 \%), 1$ penetration of the orbital cavity $(0.018 \%), 9$ cases of persistent pain $(0.16 \%)$, 5 exposures of the implant $(0.092 \%)$ and failure of osseointegration $16(0.29 \%)$. Articles of clinical cases with a small sample group reported the absence of clinical complications. The follow-up ranged from 1 to 7 years. The most frequent complication is maxillary sinusitis, and the zygomatic fixation technique may incur in numerous complications when the surgeon is inexperienced and/or do not have good anatomical knowledge of the region. There is a lack of studies with controled postoperative followup that discuss trans and postoperative complications.

Keywords: Maxillary sinusitis. Zygoma. Dental implants. 


\section{INTRODUÇÃO}

A reabilitação cirúrgica e protética do paciente desdentado tem dois objetivos principais, em primeiro lugar, a restauração da função oral e em segundo lugar a restauração da forma facia ${ }^{11}$. E por esse motivo, a reabilitação de maxilas atróficas permanece como grande desafio ao cirurgião. Nos últimos anos, as fixações zigomáticas têm sido apresentadas como opção viável para o tratamento daqueles pacientes que apresentam atrofia grave no rebordo alveolar maxilar, com extensa pneumatização dos seios maxilares, e que não desejam se submeter a procedimentos reconstrutivos ${ }^{2}$.

Quando a crista óssea residual não permite a colocação de implantes convencionais, o tratamento para a completa reabilitação de maxila gravemente atrófica pode ser realizado com 4 implantes zigomáticos e função imediata, com resultados satisfatórios em estética, função e conforto para o paciente. No entanto, mesmo que as reabilitações com implantes zigomáticos tenham mostrado uma boa taxa de sucesso, esta técnica é complexa e requer cuidados adicionais para minimizar a incidência de complicações, uma vez que são de ocorrência frequente ${ }^{3-4}$.

Pacientes com maxilas severamente reabsorvidas representam um desafio para a reabilitação protética convencional ${ }^{5}$, e os implantes zigomáticos têm se destacado como uma alternativa viável para o tratamento dos mesmos ${ }^{2}$.

O uso de implantes zigomáticos aumenta o sucesso do tratamento e diminui a utilização de enxertos ósseos e o número de etapas cirúrgicas ${ }^{6}$. Autores relatam taxa de sucesso de 97,6\%, avaliados entre 1998 e 20017-8.

Para o sucesso do tratamento, fatores como tamanho e extensão da cavidade nasal, quantidade de osso, número e tamanho dos implantes e técnica cirúrgica devem ser considerados durante o planejamento, apesar disso, alguns estudos têm relatado complicações nos tecidos moles e no seio maxilar por uso da fixação zigomática ${ }^{10-11}$.

As complicações mais comumente encontradas nos casos de fixações zigomáticas são infecções peri-implantares, sinusites crônicas, dor e saliência da fixação na região do zigoma, o que pode levar a uma falha no processo de osseointegração, ocasionando a perda desses implantes e-3,12-15. $^{2}$.

O objetivo desta revista de literatura foi identificar os estudos clínicos relevantes e ressaltar as complicações cirúrgicas relacionadas aos implantes zigomáticos nos períodos trans e pós-operatório.

\section{REVISÃO DE LITERATURA}

Esta revisão foi realizada na base de dados PubMed (US National Library of Medicine, National Institutes of Health), para o período compreendido entre 2010 e 2015, de artigos publicados em língua inglesa e que envolviam somente seres humanos. Os seguintes termos foram utilizados na estratégia de pesquisa: \{Subject AND Adjective), sendo Subject: (zygomatic OR zygoma OR zygomaticus [Title/abstract]) AND Adjective: (implant OR implants OR fixture OR fixtures [Title/abstract]).

A busca resultou em 92 artigos e, destes, foram selecionados os estudos que atendessem aos critérios (1) relatos de casos, (2) ensaios clínicos, (3) ensaios clínicos controlados, (4) ensaios-controle randomizados, (5) revisões e (6) revisões sistemáticas que incluíam pacientes com maxila atrófica que não poderiam ser reabilitados com implantes dentários convencionais devido à falta de osso, e que fossem parcial ou totalmente desdentados. Dada a escassez de artigos com notas de alto nível de evidência, os estudos com poucos relatos de caso também foram considerados para inclusão. Artigos de relato de caso sem controle pós-operatório de ao menos 1 ano e artigos de revisão sem dados originais foram excluídos, embora referências a artigos potencialmente pertinentes tenham sido anotadas para posterior análise. Com a estratégia de busca descrita foram identificados 92 estudos e, destes, selecionados 12 para avaliação.

As complicações pós-operatórias relatadas foram as seguintes: 162 casos de sinusites (2.98\%), 129 de infecção dos tecidos moles (2.37\%), 16 de parestesia $(0.29 \%), 21$ fístulas orosinusais $(0.38 \%), 2$ enfisemas subcutâneos $(0.036 \%), 1$ de penetração da cavidade orbital $(0.018 \%), 9$ de dor persistente $(0.16 \%), 5$ de exposição do implante $(0.092 \%)$ e 16 falhas de osseointegração $(0.29 \%)$. Artigos de casos clínicos com um grupo amostral pequeno relataram ausência de complicações clínicas, e o follow-up variou de 1 a 7 anos.

Nos 12 estudos selecionados foram avaliados 5.425 implantes zigomáticos instalados. A Tabela 1 resume as características de cada artigo incluído neste estudo. A Tabela 2 mostra quais as complicações encontradas e as quantidades das mesmas nos estudos incluídos nesta revisão. A Tabela 3 descreve o total das complicações destes estudos, em porcentagem. 
Tabela 1 - Características dos estudos incluídos nesta revisão.

\begin{tabular}{|c|c|c|c|c|c|c|c|c|}
\hline \multirow{2}{*}{ Autores } & \multirow{2}{*}{ Ano } & \multirow{2}{*}{$\begin{array}{c}\text { Amostra } \\
\text { (n) }\end{array}$} & \multirow{2}{*}{$\begin{array}{l}\text { Idade } \\
\text { (M) }\end{array}$} & \multirow{2}{*}{$\begin{array}{l}\text { Follow- } \\
\text { up } \\
\text { (M) }\end{array}$} & \multicolumn{4}{|c|}{ Implantes Zigomáticos } \\
\hline & & & & & 1 & $\mathrm{~F}$ & s & PF \\
\hline Bothur et al & 2015 & 14 & - & $\begin{array}{c}9,3 \\
\text { anos }\end{array}$ & 58 & 2 & $97 \%$ & - \\
\hline Maló et al. & 2015 & 352 & - & 7 anos & 747 & 7 & $94.4 \%$ & $1^{\mathrm{a}}$ ano \\
\hline $\begin{array}{l}\text { Butura e } \\
\text { Galindo }\end{array}$ & 2014 & 19 & - & - & 40 & 0 & $100 \%$ & - \\
\hline $\begin{array}{l}\text { Fernández } \\
\text { et al. }\end{array}$ & 2014 & 80 & 55,5 & 2 anos & 244 & 1 & $99.6 \%$ & - \\
\hline Goiato et al. & 2014 & 748 & - & $\begin{array}{c}42 \\
\text { meses }\end{array}$ & 1541 & 33 & $97,86 \%$ & $1^{\circ}$ ano \\
\hline Maló et al. & 2014 & 39 & 53 & 5 anos & 92 & 1 & $98.8 \%$ & 3 ano \\
\hline $\begin{array}{l}\text { Rodriguez- } \\
\text { Chessa et } \\
\text { al. }\end{array}$ & 2014 & 29 & - & $\begin{array}{c}20 \\
\text { meses }\end{array}$ & 67 & 14 & $79.1 \%$ & - \\
\hline $\begin{array}{l}\text { Chrcanovic } \\
\text { e Abreu }\end{array}$ & 2013 & 1145 & - & - & 2402 & 56 & $97.6 \%$ & $1^{\circ}$ ano \\
\hline $\begin{array}{l}\text { Davó e } \\
\text { Pons }\end{array}$ & 2013 & 17 & 57.7 & 3 anos & 68 & 0 & $100 \%$ & - \\
\hline Davo et al. & 2013 & 42 & - & 5 anos & 81 & 1 & $98.5 \%$ & $3^{\mathrm{a}}$ ano \\
\hline Yates et al. & 2013 & 25 & 64,12 & 5 anos & 43 & 6 & $86 \%$ & $1^{\circ}$ ano \\
\hline Nóia et al. & 2010 & 16 & - & - & 42 & 10 & $75 \%$ & - \\
\hline
\end{tabular}

Tabela 2 - Complicações encontradas pelos artigos incluídos nesta revisão.

\begin{tabular}{|c|c|c|c|c|c|}
\hline \multirow[b]{2}{*}{ Autores } & \multicolumn{5}{|c|}{ Complicações } \\
\hline & Sinusite & $\begin{array}{l}\text { Infecção } \\
\text { peri- } \\
\text { implantar }\end{array}$ & Parestesia & $\begin{array}{c}\text { Fistula } \\
\text { oroantral }\end{array}$ & $\begin{array}{l}\text { Perfuração } \\
\text { da cavidade } \\
\text { orbital }\end{array}$ \\
\hline Bothur S et al. & 13 & - & - & - & - \\
\hline Maló et al. & 26 & 54 & - & 1 & - \\
\hline $\begin{array}{l}\text { Butura e } \\
\text { Galindo }\end{array}$ & - & - & - & - & - \\
\hline $\begin{array}{l}\text { Fernández et } \\
\text { al. }\end{array}$ & 6 & - & 1 & 1 & 0 \\
\hline Goiato et al. & 33 & - & - & - & - \\
\hline Maló et al. & 5 & 13 & - & 1 & - \\
\hline $\begin{array}{l}\text { Rodríguez- } \\
\text { Chessa et al. }\end{array}$ & 4 & - & - & - & - \\
\hline $\begin{array}{l}\text { Chrcanovic e } \\
\text { Abreu }\end{array}$ & 70 & 48 & 15 & 17 & - \\
\hline Davó e Pons & 2 & - & - & 1 & 1 \\
\hline Davó et al. & 1 & 5 & - & 1 & - \\
\hline Yates et al. & 1 & - & - & - & - \\
\hline Nóia et al. & 1 & 4 & - & - & - \\
\hline
\end{tabular}

- Não informado pelos autores.
Tabela 3 - Prevalência das complicações descritas nos estudos avaliados.

\begin{tabular}{lc}
\hline Complicações & Prevalência \\
Sinusite & 162 casos $-2.98 \%$ \\
Fístula orosinusal & 21 casos $-0.38 \%$ \\
Enfisema subcutâneo & 02 casos $-0.036 \%$ \\
Parestesia & 16 casos $-0.29 \%$ \\
Infecção dos tecidos moles peri-implantares & 129 casos $-2.37 \%$ \\
Penetração da cavidade orbital & 01 casos $-0.018 \%$ \\
Dor persistente & 09 casos $-0.16 \%$ \\
Exposição do implante & 05 casos $-0.092 \%$ \\
Falha na osseointegração & 16 casos $-0.29 \%$ \\
\hline
\end{tabular}

\section{DISCUSSÃO}

A impossibilidade de instalação de implantes convencionais na região posterior da maxila devido à pneumatização dos seios maxilares ou à falta de espessura óssea é, atualmente, a principal indicação para a utilização das fixações zigomáticas ${ }^{16-17}$.

A reabsorção da maxila resulta muitas vezes na fusão das corticais vestibular e lingual, o que permite obtenção de boa estabilidade inicial dos implantes zigomáticos ${ }^{18-19}$.

Dentre as complicações clínicas mais citadas na instalação de implantes zigomáticos, a sinusite é prevalente $^{16,20}$.

A reação da mucosa sinusal ao implante zigomático que penetra na cavidade do seio maxilar foi estudada por Petruson $^{21}$, que seguiu casos de pacientes com uma técnica endoscópica. Em alguns casos, a mucosa estava recobrindo totalmente os implantes, enquanto que em outros casos os implantes foram parcialmente cobertos. Em uma inspeção visual, a mucosa estava normal, sem sinais de aumento da secreção ou infecção ao redor dos implantes. Mesmo após estes resultados, e considerando que a maioria das técnicas cirúrgicas de implantação transfixa o implante por meio do seio maxilar.

Qualquer patologia do seio maxilar deve, preferencialmente, ser tratada antes da instalação do implante zigomático ${ }^{12}$.

Ainda assim, outros problemas relacionados com os implantes zigomáticos foram relatados, incluindo infecções peri-implantares após a instalação implantes zigomáticos ${ }^{22-}$ ${ }^{23}$, problemas de tecido mole intra-oral ${ }^{12}$, remoção de implantes zigomáticos devido à sinusite recorrente ${ }^{3}$, formação de fístula orosinusal ${ }^{9,12}$, hematoma periorbital, lesão orbital ${ }^{22}$, déficit temporários de nervos sensoriais ${ }^{6}$, sangramento nasal moderado por 1 a 3 dias 12, e penetração 
intracraniana inadvertida com os implantes zigomáticos ${ }^{24}$.

Nos artigos revisados, as complicações encontradas foram: sinusite maxilar (2.98\%), enfisema subcutâneo malar $(0.036 \%)$, dor persistente $(0.16 \%)$, fístula orosinusal $(0.38 \%)$, infecção dos tecidos moles peri-implantares (2.37\%), parestesia do nervo infraorbital $(0.29 \%)$, penetração da cavidade orbital $(0.018 \%)$, exposição do implante $(0.092 \%)$ e falha na osseointegração com perda dos implantes $(0.29 \%)$. Em Chrcanovic e Abreu ${ }^{5}$, Goiato et al. ${ }^{14}$ e Maló et al. ${ }^{16}$, a ocorrência de sinusite foi prevalente em relação às demais complicações $(2.9 \%, 2.1 \%$ e $3.4 \%$, respectivamente).

O implante zigomático tem como característica a sua exposição ao seio maxilar, e, por isso, o controle da saúde do seio maxilar deve ser parte do controle pós-operatório. A superfície maquinada relativamente suave é preferida neste ambiente para minimizar a colonização de bactérias ${ }^{12}$. Postula-se que a conexão dos pilares definitivos no dia da cirurgia possa levar a um melhor estabelecimento da barreira de tecido mole e a um risco diminuído de comunicação orosinusal ${ }^{25-26}$.

Em relação à infecção e à formação de fístula orosinusal, especula-se que a osseointegração deficiente da parte coronal do implante zigomático seja a responsável pela complicação. O problema pode estar relacionado à alta incidência de contato entre a crista alveolar residual e o implante, criando uma comunicação entre a cavidade bucal e o seio maxilar ${ }^{12}$. Yates et al. ${ }^{17}$ não encontraram relação entre pacientes com problemas sistêmicos e/ou fumantes com falhas dos implantes zigomáticos, embora esses problemas estejam ligados a falhas de implantes convencionais, entretanto, outros estudos relatam maior prevalência de falhas no grupo tabagista ${ }^{13-14,17}$.

\section{CONCLUSÃO}

As complicações clínicas mais comuns relacionadas à instalação de implantes zigomáticos são sinusite, fístula orosinusal, infecção peri-implantar, lesão orbital, déficit nervosos sensoriais temporários, sangramento nasal moderado, dor persistente, exposição do implante e falha na osseointegração. A complicação mais frequente na utilização do implante zigomático é a sinusite maxilar, decorrente da íntima relação do implante com o seio maxilar.

A literatura acerca da utilização de implantes zigomáticos é extensa, e a maioria dos estudos incluídos nesta revisão apresentaram taxa de sobrevivência de $97.58 \%$, viabilizando a sua indicação. Entretanto, ensaios clínicos randomizados e com longo tempo de proservação ainda são necessários para testar a eficácia destes implantes no tratamento da maxila atrófica.

\section{REFERÊNCIAS}

1. Sutton DN, Lewis BR, Patel M, Cawood JI. Changes in facial form relative to progressive atrophy of the edentulous jaws. Int J Oral Maxillofac Surg. 2004;33(7):676-82.

2. Nóia CF, Ortega-Lopes R, Rodríguez-Chessa JG, Chaves-Netto HDM, Nascimento FFAO, Mazzonetto R. Complicações em fixações zigomáticas: revisão da literatura e análise retrospectiva de 16 casos. ImplantNews. 2010;7(3):381-5.

3. Al-Nawas B, Wegener J, Bender C, Wagner W. Critical soft tissue parameters of the zygomatic implant. J Clin Periodontol. 2004;31(7):497-500.

4. Mozzati M, Mortellaro C, Arata V, Gallesio G, Previgliano V. Rehabilitation with 4 zygomatic implants with a new surgical protocol using ultrasonic technique. J Craniofac Surg. 2015;26(3):722-8.

5. Chrcanovic BR, Abreu MH. Survival and complications of zygomatic implants: a systematic review. Oral Maxillofac Surg. 2013;17(2):81-93.

6. Malevez C, Abarca M, Durdu F, Daelemans P. Clinical outcome of 103 consecutive zygomatic implants: a 6-8 months follow-up study. Clin Oral Implants Res. 2004;15(1):18-22.

7. Boyes-Varley JG, Howes DG, Lownie JF, Blackbeard GA. Surgical modifications to the Brånemark zygomaticus protocol in the treatment of the severely resorbed maxilla: a clinical report. Int J Oral Maxillofac Implants. 2003;18(2):232-7.

8. Misch CE. Implantes dentários contemporâneos. 2 ed. São Paulo: Santos; 2000. p. 271-302.

9. Becktor JP, Isaksson S, Abrahamsson P, Sennerby L. Evaluation of 31 zygomatic implants and 74 regular dental implants used in 16 patients for prosthetic reconstruction of the atrophic maxilla with cross-arch fixed bridges. Clin Implant Dent Relat Res. 2005;7:159-65.

10. Brånemark PI, Grondahl K, Ohrnell LO, Nilsson P, Petrusson B, Svensson B, et al. Zygoma fixture in the management of advanced atrophy of the maxilla: technique and long-term results. Scand J Plast Reconstr Surg Hand Surg. 2004;38(2):70-85.

11. Souza JGS, Krollmann FP, Oliveira RCN. Utilização de implantes zigomáticos na reabilitação de maxilas atróficas. Rev Bras Cir Cabeça Pescoço. 2014;43(3):153-7.

12. Aparicio C, Ouazzani W, Garcia R, Arevalo X, Muela R, Fortes V. A prospective clinical study on titanium implants in the zygomatic arch for prosthetic rehabilitation of the atrophic edentulous maxilla with a follow-up of 6 months to 5 years. Clin Implant Dent Relat Res. 2006;8(3):114-22.

13. Galán Gil S, Peñarrocha Diago M, Balaguer Martínez J, Marti Bowen E. Rehabilitation of severely resorbed maxillae with zygomatic implants: an update. Med Oral Patol Oral Cir Bucal. 2007;12(3):E216-20.

14. Goiato MC, Pellizzer EP, Moreno A, Gennari-Filho H, dos Santos DM, Santiago JF Jr, et al. Implants in the zygomatic bone for maxillary prosthetic rehabilitation: a systematic review. Int J Oral Maxillofac Surg. 2014;43(6):748-57

15. Pi Urgell J, Revilla Gutiérrez V, Gay Escoda CG. Rehabilitation of atrophic maxilla: a review of 101 zygomatic implants. Med Oral 
Patol Oral Cir Bucal. 2008;13(6):E363-70.

16. Maló P, Nobre M, Lopes A, Ferro A, Moss S. Extramaxillary surgical technique: clinical outcome of 352 patients rehabilitated with 747 zygomatic implants with a follow-up between 6 months and 7 years. Clin Implant Dent Relat Res. 2015;17 Suppl 1:e153-62.

17. Yates JM, Brook IM, Patel RR, Wragg PF, Atkins SA, El-Awa A, et al. Treatment of the edentulous atrophic maxilla using zigomatic implants: evaluation of survival rates over 5-10 years. Int J Oral Maxillofac Surg. 2014;43(2):237-42.

18. Migliorança RM, Ilg JP, Mayo TM, Serrano AS, Funis LP, Souza RP. Função imediata em fixações zigomáticas: relato de dois casos com 18 e 30 meses de acompanhamento clínico. Implantnews. 2006;3(3):243-7.

19. Saad PA, Jorge R, Faloppa F, Bottino MA, Vasconcellos DK. Fixações zigomáticas Zigomax: novos conceitos e proposta de um novo design. ImplantNews 2005;2(5):499-502.

20. Fernández H, Gómez-Delgado A, Trujillo-Saldarriaga S, VarónCardona D, Castro-Núñez J. Zygomatic implants for the management of the severely atrophied maxilla: a retrospective analysis of 244 implants. J Oral Maxillofac Surg. 2014;72(5):887-91.

21. Petruson B. Sinuscopy in patients with titanium implants in the nose and sinuses. Scand J Plast Reconstr Surg Hand Surg. 2004;38(2):86-93.

22. Davó R, Pons O. Prostheses supported by four immediately loaded zygomatic implants: a 3-year prospective study. Eur J Oral Implantol. 2013;6(3):263-9.

23. Maló P, Nobre M, Lopes A, Ferro A, Moss S. Five-year outcome of a retrospective cohort study on the rehabilitation of completely edentulous atrophic maxillae with immediately loaded zygomatic implants placed extra-maxillary. Eur J Oral Implantol. 2014;7(3):267-81

24. Reychler H, Olszewski R. Intracerebral penetration of a zygomatic dental implant and consequent therapeutic dilemmas: case report. Int J Oral Maxillofac Implants. 2010;25(2):416-8.

25. Davó R, Malevez C, Pons O. Immediately loaded zygomatic implants: a 5-year prospective study. Eur J Oral Implantol. 2013;6(1):39-47.

26. Gonçalves LM, Gonçalves TM, Rodrigues AH, Lanza MD, do Nascimento PR, Girundi FM. Intra-and extraoral prostheses retained by zygoma implants following resection of the upper lip and nose. J Prosthodont. 2015;24(2):172-7.

27. Bothur S, Kullendorff B, Olsson-Sandin G. Asymptomatic chronic rhinosinusitis and osteitis in patients treated with multiple zygomatic implants: a long-term radiographic follow-up. Int J Oral Maxillofac Implants. 2015;30(1):161-8.

28. Butura CC, Galindo DF. Combined immediate loading of zygomatic and mandibular implants: a preliminary 2-Year report of 19 patients. Int J Oral Maxillofac Implants. 2014;29(1):e22-9.

29. Rodríguez-Chessa JG, Olate S, Netto HD, Shibli J, de Moraes M, Mazzonetto R. Treatment of atrophic maxilla with zygomatic implants in 29 consecutives patients. Int J Clin Exp Med. 2014;7(2):426-30. 